\title{
Produção da toxina Cry1Ac e preferência para alimentação e oviposição de Alabama argillacea em algodão Bt sob estresse hídrico
}

\author{
Mauricio Silva de Lima(1) e Jorge Braz Torres(2)
}

\begin{abstract}
(1)Universidade Federal de Alagoas, Centro de Ciências Agrárias, Campus Delza Gitaí, BR101-Norte, Km 85, CEP 57100-000 Rio Largo, AL. E-mail: mauriciosilvalima@gmail.com (2)Universidade Federal Rural de Pernambuco, Departamento de Agronomia, Avenida Dom Manoel de Medeiros, s/no, Dois Irmãos, CEP 52171-900 Recife, PE. E-mail: jtorres@depa.ufrpe.br
\end{abstract}

Resumo - O objetivo deste trabalho foi avaliar a produção da toxina Cry1Ac de Bacillus thuringiensis (Bt) em plantas de algodão geneticamente modificado (algodão $\mathrm{Bt}$ ), e a preferência para alimentação e oviposição de Alabama argillacea confinada em algodão $\mathrm{Bt}$ e não $\mathrm{Bt}$ sob estresse hídrico. As variedades de algodão $\mathrm{Bt}-\mathrm{Acala}$ 90B e NuOpal - e não Bt - Acala 90 e DeltaOpal - foram cultivadas em microparcelas, com e sem estresse hídrico. A produção de toxina foi avaliada em folhas e partes reprodutivas das variedades de algodão Bt. Para o teste de escolha para oviposição, mariposas de A. argillacea foram liberadas em telado que continha as quatro variedades do algodão. Após 72 horas, o número de ovos foi quantificado. Para a preferência de alimentação, foram avaliadas lagartas com três e 10 dias de idade. A produção da toxina Cry1Ac foi superior nas folhas da parte apical das plantas, das duas variedades sob estresse hídrico, e similar nas brácteas e cascas de maçãs. Lagartas e mariposas de $A$. argillacea não diferenciam algodão Bt e não Bt na escolha quanto à alimentação e à oviposição, respectivamente. Entretanto, as mariposas preferem ovipositar em plantas sem estresse hídrico.

Termos para indexação: Bacillus thuringiensis, Gossypium hirsutum, curequerê-do-algodoeiro, herbivoria, resistência de plantas.

\section{Cry1Ac toxin production and feeding and oviposition preference of Alabama argillacea in Bt cotton under water stress}

\begin{abstract}
The objective of this work was to evaluate the production of Cry1Ac, a toxin of Bacillus thuringiensis (Bt), in genetically modified cotton plants (Bt cotton), and the feeding and oviposition preference of Alabama argillacea confined to Bt cotton and non Bt cotton under water stress. The varieties of Bt cotton - Acala 90B and NuOpal, and non $\mathrm{Bt}$ - Acala 90 and DeltaOpal - were cultivated in microplots, under stress and no stress conditions. Toxin production was evaluated in Bt cotton leaves and reproductive parts. For the oviposition choosing test, A. argillacea moths were released into a large screen cage which contained the four cotton varieties. After 72 hours, the number of eggs was quantified. For the feeding preference, 3-day and 10-day-old larvae were evaluated. The production of Cry1Ac toxin was higher in leaves of the plant apical part of the two varieties under water stress, and it was similar in the bracts and boll husks. Moths and caterpillars of A. agillacea do not distinguish between Bt and non Bt cotton for feeding and oviposition choosing, respectively. However, moths show preference for ovipositing on plants under no water stress.
\end{abstract}

Index terms: Bacillus thuringiensis, Gossypium hirsutum, cotton leafworm, herbivory, plant resistance.

\section{Introdução}

O algodoeiro é hospedeiro de um complexo de lepidópteros que desfolham as plantas ou destroem partes reprodutivas como os botões florais e maçãs. $\mathrm{O}$ algodão geneticamente modificado para a produção de toxinas Cry do Bacillus thuringiensis, algodão Bt, é eficaz no controle do curuquerê-do-algodoeiro [Alabama argillacea (Hübner)], lagartas-das-maçãs [Heliothis virescens (Fabricius) e Helicoverpa zea
(Boddie) (Lep.: Noctuidae)] e da lagarta-rosada [Pectinophora gossypiella (Saunders) (Lep.: Gelechiidae)], espécies consideradas pragas-chave da cultura no Cerrado e Semiárido do Brasil. Entre essas espécies, A. argillacea pode ocorrer da fase inicial à maturação da lavoura e em todas as regiões cotonicultoras do Brasil (Soares \& Silva, 2003) e, frequentemente, ocasiona danos de desfolha que justificam seu controle químico (Domiciano \& Santos, 1994). 
Alabama argillacea é específica do continente americano e é conhecida como praga apenas em algodoeiro e, portanto, estará exposta continuamente à toxina Cry produzida pelo algodão $\mathrm{Bt}$, durante toda a safra. A monofagia em lepidópteros, conforme a apresentada por $A$. argillacea, os condiciona a localizar e selecionar o alimento por meio de pistas visuais, químicas e gustatórias (Papaj \& Rausher, 1983). Espécies com preferência hospedeira devem ser capazes de detectar pequenas mudanças na qualidade da planta, seja por elementos voláteis emitidos ou estrutura física do substrato onde a oviposição é feita, para aumentar o sucesso da sua descendência (Zalucki et al., 2002). Assim, um possível reconhecimento da planta de algodão $\mathrm{Bt}$ pela mariposa de $A$. argillacea, para oviposição, pode influenciar o processo de seleção de populações do inseto; e no manejo da praga pelo uso do algodão Bt será fundamental a utilização da estratégia de áreas de refúgio com algodão não $\mathrm{Bt}$, para retardar a seleção de populações resistentes (Jongsma et al., 2010). Vários estudos com lepidópteros indicam uma relação positiva entre o melhor desempenho da fase imatura e a seleção futura do hospedeiro quanto à oviposição (Gripenberg et al., 2010). Apesar desses estudos, poucos foram realizados com mariposas e, em especial, com espécies praga de importância econômica. Resultados com Helicoverpa armigera (Hübner) e Trichopluisa ni (Hübner) mostram que há correlação entre o desempenho da fase larval e o condicionamento prévio de adultos na preferência quanto à oviposição (Cunningham et al., 1998; Shikano et al., 2010).

As variedades de algodão $\mathrm{Bt}$, conhecido nas Américas como algodão Bollgard, disponíveis atualmente para plantio comercial no Brasil, são Acala 90B, NuOpal e DP 604B, e todas produzem constitutivamente a toxina Cry1Ac. Em virtude da complexidade do controle de pragas do algodoeiro, pela existência de inúmeras espécies de pragas-chave, e do uso intensivo de inseticidas para o controle delas, a facilidade de adoção do plantio do algodão Bt tem sido uma das razões de sua utilização por pequenos produtores da África do Sul (Bennett et al., 2006b), México (Traxler \& Godoy-Avila, 2004), China (Pray et al., 2004) e Índia (Bennett et al., 2006a). Similarmente, o plantio do algodão no Semiárido do Nordeste é feito basicamente por pequenos produtores, em áreas entre 0,5 e 8 ha (Fontes et al., 2006), e o cultivo do algodão Bt pode ser uma alternativa viável. Entretanto, nessa região, as plantas estão sujeitas à condição de estresse hídrico, em consequência de períodos de veranico ou mesmo da redução de precipitação durante a safra.

Fatores ambientais como fertilidade e umidade do solo podem influenciar a produção da toxina Cry na planta (Adamczyk Junior \& Sumerford, 2001; Torres et al., 2009). A produção da toxina varia ao longo do desenvolvimento e nas diferentes partes da planta de algodão (Adamczyk Junior \& Sumerford, 2001; Torres et al., 2006). Essa variabilidade pode, entretanto, comprometer a eficácia da tecnologia, apesar do alto grau de suscetibilidade de lagartas de $A$. argillacea ao algodão Bt (Santos \& Torres, 2010; Sousa et al., 2010). Todavia, a monofagia de A. argillacea em algodoeiro $\mathrm{e} o$ alto grau de suscetibilidade às toxinas Cry podem favorecer a pressão de seleção sobre as populações do inseto, com consequente alteração de comportamento da seleção do hospedeiro (Papaj \& Rausher, 1983; Jongsma et al., 2010).

O Brasil está iniciando o plantio em larga escala de variedades de algodão Bt. Na safra 2009, em seu quarto ano após a liberação comercial, o Brasil plantou 145 mil hectares de algodão Bt (James, 2009), com grande expectativa de aumento de área cultivada com a liberação de novas variedades, com diferentes genes para a resistência a insetos e herbicidas.

O objetivo deste trabalho foi avaliar a produção da toxina Cry1Ac de Bacillus thuringiensis (Bt) em plantas de algodão geneticamente modificado (algodão $\mathrm{Bt}$ ), e a preferência para alimentação e oviposição de Alabama argillacea, confinada em algodão Bt e nãoBt sob estresse hídrico.

\section{Material e Métodos}

Os experimentos foram realizados na Área de Fitossanidade, do Departamento de Agronomia da Universidade Federal Rural de Pernambuco (UFRPE), em microparcelas e telados, e no Laboratório de Ecologia e Controle Biológico da UFRPE.

A espécie $A$. argillacea foi obtida da criação de manutenção do laboratório e alimentada com folhas de algodão da variedade BRS Verde, durante a fase larval, e solução de mel a $10 \%$ durante a fase adulta, segundo a metodologia descrita em Santos et al. (2008). 
Quatro variedades de algodão, duas transgênicas (Bt) - Acala 90B e NuOpal - e duas isolinhas não transgênicas (não-Bt) - Acala 90 e DeltaOpal - foram cultivadas em dois telados, com seis microparcelas cada uma, constituídas de anéis de cimento de 1,0 m de diâmetro e $0,5 \mathrm{~m}$ de altura, preenchidos com solo fertilizado de acordo com as exigências do algodoeiro. Em cada microparcela, foram cultivadas quatro plantas de algodão de cada variedade, equidistantes $30 \mathrm{~cm}$ entre si. Na parte central da microparcela, foram instalados tensiômetros de pulsão analógicos (SondaTerra Equipamentos Agronômicos, Piracicaba, SP), para monitorar a umidade do solo. As plantas foram cultivadas em condições ideais de umidade do solo $(0,2$ a 0,4 psi) até o início da floração e, a partir dessa fase, a irrigação das seis microparcelas sob estresse hídrico foi mantida à pressão média de 0,6 a 0,8 psi. As medições foram feitas regularmente, no mesmo horário pela manhã e, quando necessário, foi realizada a reposição de água para obtenção da umidade adequada do solo.

Para avaliar a produção da toxina Cry1Ac, em algodão $\mathrm{Bt}$, utilizou-se o delineamento experimental inteiramente casualizado, em arranjo fatorial $2 \times 2$, duas condições hídricas (com e sem estresse hídrico) e duas variedades de algodão $\mathrm{Bt}$ (Acala 90B e NuOpal), com seis repetições. As amostras do limbo foliar das duas variedades foram coletadas da folha expandida mais alta do topo das plantas, tendo-se utilizado microtubos de $2 \mathrm{~mL}$, aos $0,16,26,32$ e 47 dias após o estresse hídrico. A folha foi dobrada ao longo da nervura principal e, com a tampa do tubo, cortou-se um disco foliar, com o mesmo diãmetro do tubo, e que foi depositado em seu interior. Assim, a folha ficava marcada pelo orifício deixado em cada coleta, o que facilitava a localização para as coletas posteriores. Partes reprodutivas das plantas (pétalas, brácteas de botões florais e cascas de maçãs macias) foram coletadas aos 14 dias após o estresse hídrico.

As amostras foram congeladas a $-20^{\circ} \mathrm{C}$, até $\mathrm{o}$ momento da extração e quantificação da toxina. A extração foi feita em triplicata, com $100 \mathrm{mg}$ de massa de matéria fresca em solução "phosphate buffered saline + Tween 20" (PBST) na diluição de 1:10 (p/v). A quantificação foi feita com kits para Elisa PathoScreen (Agdia Inc., Elkhart, IN, EUA). Acompanhada do kit, foi adquirida a toxina Cry1Ac purificada, à concentração de $40 \mathrm{ng}$, e foram realizadas diluições de 40 a 0,172 ng para a construção da curva padrão de detecção da concentração da toxina. A condução do teste de Elisa, leitura e interpretação dos resultados seguiram a metodologia descrita em Torres \& Ruberson (2008).

Os dados de concentração de Cry1Ac foram submetidos ao teste de normalidade e homogeneidade de variância, pelo Proc Univariate do SAS (SAS Institute, 2001), e transformados, quando necessário. Em seguida, foram submetidos à análise de variância, e as médias foram comparadas pelo teste de Fisher da ANOVA.

Para o teste de escolha de oviposição, dez mariposas de $A$. argillacea, no início da escotófase, foram colocadas em cada telado com as quatro variedades de algodão, cultivadas nas seis microparcelas, três sob estresse hídrico (20 dias de estresse hídrico) e três sem estresse (repetições controle). Assim, 24 plantas de algodoeiro $\mathrm{Bt}$ ou não $\mathrm{Bt}$, sob estresse hídrico ou não, foram expostas à oviposição das mariposas, por telado. $\mathrm{O}$ experimento foi repetido duas vezes.

Após 72 horas da liberação das mariposas, o número de ovos depositados por planta foi quantificado, e as mariposas sobreviventes foram coletadas e descartadas. O número de ovos depositado por planta foi submetido à análise de frequência, para o teste de igualdade de escolha de oviposição das mariposas, entre as quatro plantas cultivadas por parcela, por meio do PROC FREQ do SAS (SAS Institute, 2001), e interpretado pelo teste de qui-quadrado.

Para a avaliação da preferência de alimentação entre plantas Bt e não-Bt, foram utilizadas lagartas de A. argillacea com três e dez dias de idade.

Discos de folhas com $5 \mathrm{~cm}$ de diâmetro, das quatro variedades, foram coletados da parte mediana do limbo foliar de folhas expandidas do topo da planta, com um vasador circular. As folhas colhidas foram previamente lavadas em água corrente e deixadas para que secasse o excesso de água, porém com o pecíolo imerso em frasco com água para mantê-las túrgidas.

Foram utilizadas arenas circulares de $40 \mathrm{~cm}$ de diâmetro e $6 \mathrm{~cm}$ de altura e com dez compartimentos de escolha. Em cada arena, dois discos de folhas de cada variedade foram dispostos, individualmente, em oito compartimentos ao acaso, sendo que dois compartimentos foram deixados sem alimento para a escolha. O experimento foi conduzido em delineamento de múltipla escolha com quatro tratamentos com 
20 arenas (repetições), sendo considerado como média da repetição, o número médio de lagartas presentes nos dois discos de cada variedade oferecidos por arena. No momento da deposição dos discos foliares nos compartimentos da arena, foi construído um mapa correspondente às variedades, para possibilitar a avaliação do número de lagartas que realizavam a escolha para a alimentação, nos intervalos de 2, 4 e 24 horas após a liberação. Em seguida, na parte central da arena, foram liberadas dez lagartas do curuquerê, que foram monitoradas quanto à escolha.

No experimento com lagartas aos três dias de idade, uma das repetições foi perdida em razão do escape de lagartas para a avaliação de 24 horas. $\mathrm{O}$ número de lagartas presentes em cada disco de folha/variedade foi registrado e, posteriormente, transformado em percentagem de escolha, em função do total de lagartas utilizadas. Em seguida, os dados foram submetidos ao PROC FREQ do SAS (SAS Institute, 2001), para testar a hipótese de igualdade de escolha de $25 \%$ entre as variedades (100\% entre as quatro variedades) e interpretação pelo teste de qui-quadrado, a $5 \%$ de probabilidade.

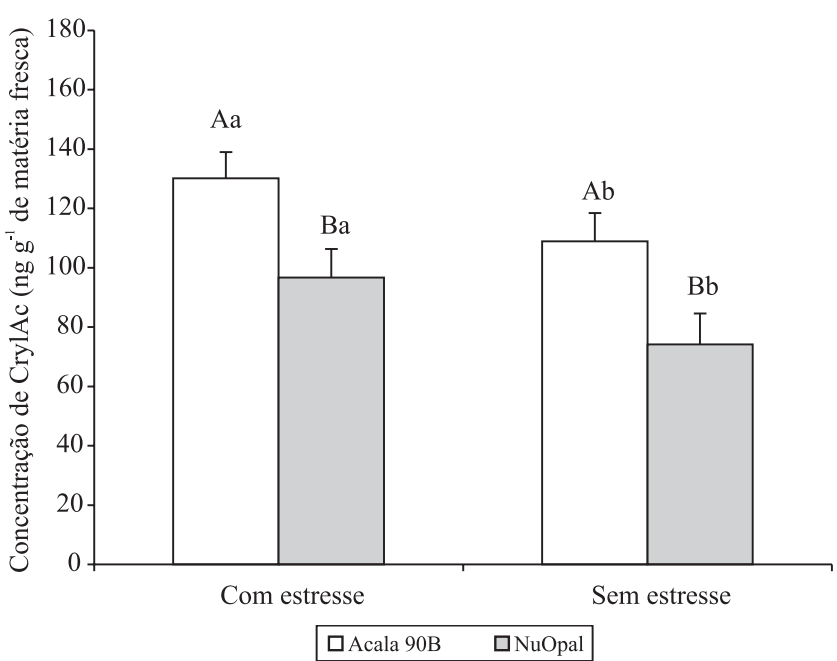

Figura 1. Concentração de Cry1 Ac (ng g-1 de matéria fresca), em folhas desenvolvidas do topo de plantas de algodão $\mathrm{Bt}$ Acala 90B e NuOpal, em quatro coletas (16 aos 47 dias), após estresse hídrico controlado. Médias \pm erro-padrão seguidas de letras iguais, minúsculas para a mesma variedade, entre as condições com e sem estresse hídrico, e maiúsculas para a mesma condição hídrica, entre as variedades, não diferem entre si, pelo teste $\mathrm{F}$, a $5 \%$ de probabilidade.

\section{Resultados e Discussão}

A produção de CrylAc em folhas expandidas do topo das plantas de algodão $\mathrm{Bt}$ variou em consequência das variedades estudadas $(\mathrm{p}<0,05)$, bem como da condição hídrica $(\mathrm{p}<0,01)$ (Figura 1). A resposta das plantas foi similar, em ambas as condições hídricas, e não houve interação significativa entre as variedades e as condições com e sem estresse hídrico.

Em relação às partes reprodutivas das plantas avaliadas, foi encontrada maior concentração da toxina nas pétalas, seguida pelas brácteas e cascas das maçãs macias (Tabela 1). Não foi possível detectar a toxina CrylAc em cascas de maçãs da variedade Acala 90B sob estresse hídrico. A concentração de Cry1Ac foi superior nas brácteas de botões florais e em folhas do topo das plantas de Acala 90B, em comparação às brácteas (Tabela 1) e às folhas de NuOpal (Figura 1).

Entre as condições hídricas, foi observada maior concentração da toxina em folhas de plantas sob estresse hídrico, em ambas as variedades (Figura 1). A maior concentração da toxina Cry1Ac nas folhas, em relação às demais partes das plantas, era esperada, pois a toxina é predominantemente expressa em tecidos verdes da planta e de forma constitutiva (Purcell et al., 2004).

Em geral, a concentração de Cry1Ac, em folhas e demais partes reprodutivas das plantas, esteve dentro da faixa de variação detectada em outros estudos, com outras variedades de algodão Bt (Adamczyk Junior \& Sumerford, 2001; Torres et al., 2006) e foi compatível com as concentrações letais de Cry1Ac, determinadas para espécies pragas do algodoeiro, como

Tabela 1. Concentração de CrylAc (ng g-1 de matéria fresca), em brácteas de botão floral, pétalas e cascas de maçã macia, das variedades de algodão Bt Acala 90B e NuOpal, aos 14 dias após as plantas terem sido submetidas ou não ao estresse hídrico controlado ${ }^{(1)}$.

\begin{tabular}{lccc}
\hline Condição hídrica & Parte da planta & NuOpal & Acala 90B \\
\hline \multirow{2}{*}{ Sem estresse } & Brácteas & $9,0 \pm 5,50 \mathrm{a}$ & $29,0 \pm 1,52 \mathrm{~b}^{*}$ \\
& Pétalas & $48,0 \pm 1,00 \mathrm{a}$ & $47,3 \pm 1,54 \mathrm{a}$ \\
& Cascas das maçãs & $10,8 \pm 8,60 \mathrm{a}$ & $5,1 \pm 3,51 \mathrm{a}$ \\
\hline \multirow{3}{*}{ Com estresse } & Brácteas & $18,0 \pm 0,00 \mathrm{a}$ & $36,3 \pm 0,66 \mathrm{~b}^{*}$ \\
& Pétalas & $35,6 \pm 2,02 \mathrm{a}$ & $46,0 \pm 2,88 \mathrm{a}$ \\
& Cascas das maçãs & $5,8 \pm 1,14$ & $-\mathbf{-}^{(2)}$ \\
\hline
\end{tabular}

${ }^{(1)}$ Médias \pm erro-padrão seguidas de letras iguais, nas colunas, em condições com e sem estresse hídrico, não diferem pelo teste F, a 5\% de probabilidade. *Médias \pm erro padrão diferem, nas linhas, a 5\% de probabilidade. ${ }^{(2)}$ Toxina Cry1Ac não detectada na concentração mínima testada de $0,172 \mathrm{ng}$. 
a lagarta-rosada e a lagarta-da-maçã (Perlak et al., 2001). Embora não se tenha linha base de suscetibilidade para A. argillacea, esta espécie se mostra altamente suscetível à toxina Cry1Ac (Santos \& Torres, 2010; Sousa et al., 2010).

A variação na produção da toxina Cry em plantas Bt pode comprometer o desempenho do controle das pragas-alvo. A variação pode ocorrer ao longo da estação, de acordo com: a idade da planta (Adamczyk Junior \& Sumerford, 2001; Torres et al., 2006); a parte da planta; a variedade transformada; e o local de plantio, em razão das condições edafoclimáticas (Adamczyk Junior \& Sumerford, 2001; Perlak et al., 2001). Os resultados do presente trabalho indicam que, apesar da diferença observada em consequência do estresse hídrico, esta condição não causou a redução na concentração da toxina, como esperado, bem como não causou diferença significativa na concentração da toxina, entre as diferentes partes das plantas, exceto nas folhas do topo das plantas onde já se esperava uma maior concentração de CrylAc.

Assim, os resultados obtidos no presente trabalho, quanto à produção de Cry1Ac sob as condições experimentais de estresse hídrico, são importantes e, ainda, fornecem a base de dados para futuro monitoramento da produção desta toxina em estudos comparativos entre regiões, variedades e condições de cultivo. Os resultados mostram também que, mesmo sob condição de estresse hídrico, a produção de Cry1 Ac não é afetada, e que o controle do curuquerê continua efetivo mesmo nessas condições, resultados estes que corroboram os de Santos \& Torres (2010).

Mariposas de $A$. argillacea apresentaram variação quanto à escolha para oviposição entre as plantas das variedades $\mathrm{Bt}$ e não $\mathrm{Bt}$ e condição hídrica. Independentemente das variedades Bt e não $\mathrm{Bt}$, houve menor oviposição em plantas sob estresse hídrico $(\mathrm{p}<0,001)$ (Figura 2).

Plantas sob estresse hídrico apresentam redução de umidade, amido e carboidratos, como também aumento na concentração de nitrogênio e de açúcares solúveis em suas folhas. Alterações nutricionais e morfológicas, em plantas sob estresse hídrico, são tidas como responsáveis por favorecer o aumento populacional de insetos herbívoros, em especial, insetos sugadores (Mattson \& Haack, 1987; McQuate \& Connor, 1990). As mariposas de A. argillacea parecem responder de forma diferente e reconhecer tais modificações, pois preferem ovipositar em folhas de plantas sem estresse hídrico. Sabe-se que mariposas são capazes de reconhecer e depositar ovos em hospedeiros mais favoráveis ao desenvolvimento de sua descendência (Papaj \& Rausher, 1983). De fato, a hipótese do estresse hídrico associado a surtos populacionais de insetos sugadores de seiva fundamenta-se na menor diluição de nutrientes na seiva, que a torna mais concentrada em plantas sob estresse. Além disso, os insetos mastigadores consomem todo o material e, portanto, podem sofrer mais com a redução da turgidez e do aumento da resistência física do material.

Mariposas de $A$. argillacea não mostraram capacidade de reconhecer e variar a oviposição entre plantas Bt e não-Bt, independentemente da condição hídrica avaliada. O curuquerê é uma praga considerada monófaga e estará constantemente submetida à toxina produzida pela planta de algodão Bt durante toda a safra. No entanto, salienta-se que a população de mariposas utilizadas no presente trabalho não teve contato, em gerações anteriores, com plantas de algodão Bt. Como a utilização de plantas transgênicas é relativamente recente no Brasil podemos considerar a hipótese de que ainda não houve tempo suficiente para permitir a seleção de mariposas capazes de reconhecer o hospedeiro adequado (Bernays \& Wcislo, 1994; Reitz \& Trumble, 1998; Jongsma et al., 2010).

Os resultados semelhantes de oviposição de A. argillacea, em algodão $\mathrm{Bt}$ e não $\mathrm{Bt}$, corroboram os de densidades de ovos das lagartas-das-maçãs, $H$. virescens e H. zea, amostrados em campos de algodão Bt e não Bt, durante três anos (Torres \& Ruberson, 2006). Este comportamento de $A$. argillacea, de não distinção entre plantas Bt e não Bt para oviposição, pode ser explicado

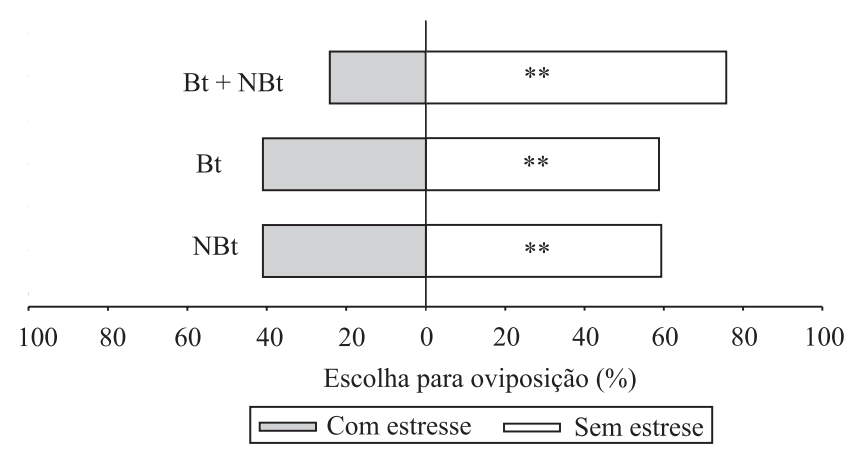

Figura 2. Proporção média de ovos de Alabama argillacea, depositados nas variedades de algodão $\mathrm{Bt}(\mathrm{Bt})$ e não $\mathrm{Bt}$ (NBt) submetidas ou não a estresse hídrico $(n=1.285$ ovos avaliados). **Significativo a $1 \%$ de probabilidade. 
pelo fato de que, à exceção da produção constitutiva da toxina na planta, não há outra diferença fenotípica entre as variedades Bt e não Bt. Em especial, para espécies monófagas como $A$. argillacea, que não dispõe de plantas alternativas para oviposição (Tate \& Lewis, 2006).

Lagartas de três ou 10 dias de idade não mostraram preferência para se alimentar em folhas de algodão $\mathrm{Bt}$ ou não $\mathrm{Bt}$, ao se contrastar simultaneamente as quatro variedades de algodão testadas. Uma diferenciação na alimentação, em especial de lagartas maiores do que as testadas neste trabalho (10 dias de idade), poderia resultar em maior pressão de seleção de populações para a resistência. Primeiro, as lagartas maiores poderiam se dispersar para uma planta mais próxima não $\mathrm{Bt}$, em casos de mistura de sementes, ou para estruturas de menor concentração da toxina, como brácteas de botões florais, ou se alimentar em cascas de maçãs (Tabela 1), embora não sejam alimentos preferidos pelas lagartas de A. argillacea, em comparação às folhas (Quirino \& Soares, 2001).

O comportamento de escolher o alimento, pelas lagartas, na exposição de plantas Bt, tem sido considerado importante para decisões de manejo, considerando-se a produção da toxina na planta e os locais de expressão desejados na planta de acordo com hábito alimentar (Gore et al., 2002). No presente trabalho, foi observado que as lagartas de $A$. argillacea com três ou 10 dias de idade não apresentaram comportamento de escolha de alimento, após se alimentarem de folhas de algodão Bt (Cry1Ac), entre duas e 24 horas de observações, bem como adultos para oviposição.

\section{Conclusões}

1. O estresse hídrico das plantas não afeta a produção da toxina Cry1 Ac em algodoeiro Bt.

2. Mariposas de Alabama argillacea não diferenciam algodão Bt e não Bt para oviposição, mas diferenciam plantas de algodão sob estresse hídrico e preferem ovipositar em plantas sem estresse hídrico.

3. Lagartas de A. argillacea não diferenciam algodão Bt e não Bt para alimentação.

\section{Agradecimentos}

À Coordenção de Aperfeiçoamento de Pessoal de Nível Superior, por concessão de bolsa; ao
Conselho Nacional de Desenvolvimento Científico e Tecnológico e ao projeto Rede de Diagnóstico e Ações Multidisciplinares para a Sustentabilidade do Algodão em Pernambuco, pelo suporte financeiro.

\section{Referências}

ADAMCZYK JUNIOR, J.J.; SUMERFORD, D.V. Potential factors impacting season-long expression of Cry1 Ac in 13 commercial varieties of Bollgard cotton. Journal of Insect Science, v.1, p.1-6, 2001.

BENNETT, R.; KAMBHAMPATI, U.; MORSE, S.; ISMAEL, Y. Farm-level economic performance of genetically modified cotton in Maharashtra, India. Review of Agricultural Economics, v.28, p.59-71, 2006a.

BENNETT, R.; MORSE, S.; ISMAEL, Y. The economic impact of genetically modified cotton on South African smallholders: yield, profit and health effects. Journal of Development Studies, v.42, p.662-677, 2006b.

BERNAYS, E.A.; WCISLO, W.T. Sensory capabilities, information processing and resource specialization. The Quaterly Review of Biology, v.69, p.187-204, 1994.

CUNNINGHAM， J.P.; JALLOW， M.F.A.; WRIGHT， D.J.; ZALUCKI, M.P. Learning in host selection in Helicoverpa armigera (Hübner) (Lepidoptera: Noctuidae). Animal Behavior, v.55, p.227-234, 1998.

DOMICIANO, N.L.; SANTOS, W.J. dos. Momento adequado para aplicação de inseticida no controle do curuquerê-do-algodoeiro Pesquisa Agropecuária Brasileira, v.29, p.7-11, 1994.

FONTES, E.M.G.; RAMALHO, F.S.; UNDERWOOD, E.; BARROS, P.A.V.; SIMON, M.F.; SUJII, E.R.; PIRES, C.S.S.; BELTRÃO, N.; LUCENA,W.A.; FREIRE, E.C. The cotton agricultural context in Brazil. In: HILBECK, A.; ANDOW, D.A.; FONTES, E.M.G. (Ed.). Environmental risk assessment of genetically modified organisms: methodologies for assessing $\mathrm{Bt}$ cotton in Brazil. Wallingford: CABI, 2006. p.21 66.

GORE, J.; LEONARD, B.R.; CHURCH, G.E.; COOK, D.R. Behavior of bollworm (Lepidoptera: Noctuidae) larvae on genetically engineered cotton. Journal of Economic Entomology, v.95, p.763-769, 2002.

GRIPENBERG, S.; MAYHEW, P.J.; PARNELL, M.; ROSLIN, T. A meta-analysis of preference-performance relationships in phytophagous insects. Ecology Letters, v.13, p.383-393, 2010.

JAMES, C. Global status of commercialized Biotech/GM Crops: 2009. Ithaca: The International Service for the Acquisition of Agri-biotech Applications, 2009. (ISAAA. Brief, 41).

JONGSMA, M.A.; GOULD, F.; LEGROS, M.; YANG, L.; VAN LOON, J.J.A.; DICKE, M. Insect oviposition behavior affects the evolution of adaptation to Bt crops: consequences for refuge policies. Evolution Ecology, v.24, p.1017-1030, 2010.

MATTSON, W.J.; HAACK, R.A. The role of drought in outbreaks of plant-eating insects. Bioscience, v.37, p.110-118, 1987. 
MCQUATE, G.T.; CONNOR, E.F. Insect responses to plant water deficits. 1: Effect of water deficits in soybean plants on the feeding preference of Mexican bean beetle larvae. Ecological Entomology, v.15, p.419-431, 1990.

PAPAJ, D.R.; RAUSHER, M.D. Individual variation in host location by phytophagous insects. In: AHMAD, S. (Ed.). Herbivorous insects: host-seeking behavior and mechanisms. New York: Academic Press, 1983. p.77-124.

PERLAK, F.J.; OPPENHUIZEN, M.; GUSTAFSON, K.; VOTH, R.; SIVASUPRAMANIAM, S.; HEERING, D.; CAREY, B.; IHRIG, R.A.; ROBERTS, J.K. Development and commercial use of Bollgard cotton in the USA: early promises versus today's reality. The Plant Journal, v.6, p. 489-501, 2001.

PRAY, C.; HUANG, J.; HU, R.; ROZELLE, S. Five years of Bt cotton in China - the benefits continue. The Plant Journal, v.31, p.423-430, 2004.

PURCELL, J.P.; OPPENHUIZEN, M.; WOFFORD, T.; REED, A.J.; PERLAK, F.J. The story of Bollgard cotton. In: CHRISTOU, P.; KLEE, H. (Ed.). Handbook of plant biotechnology. Indiana: Wiley, 2004. p.1148-1163.

QUIRINO, E. da S.; SOARES, J.J. Efeito do ataque de Alabama argillacea no crescimento vegetativo e sua relação com a fenologia do algodoeiro. Pesquisa Agropecuária Brasileira, v.36, p.1005-1010, 2001

REITZ, S.R.; TRUMBLE, J.T. Potential impact of larval behavior on the use of transgenic crops expressing Bacillus thuringiensis. IOBC Bulletin, v.21, p.229-233, 1998.

SANTOS, R.L.; TORRES, J.B. Produção da proteína Cry1Ac em algodão transgênico e controle de lagartas. Revista Brasileira de Ciências Agrárias, v.5, p.509-517, 2010

SANTOS,R.L.dos;TORRES,J.B.;BASTOS,C.S.Desenvolvimento e crescimento populacional de Alabama argillacea em algodoeiro de fibra branca e colorida. Pesquisa Agropecuária Brasileira, v.43, p.457-463, 2008.

SAS INSTITUTE. SAS/STAT user's guide. Version 8.02. Cary: SAS Institute, 2001.

SHIKANO, I.; AKHTAR, Y.; ISMAN, M.B. Relationship between adult and larval host plant selection and larval performance in the generalist moth, Trichoplusia ni. Arthropod-Plant Interactions, v.4, p.197-205, 2010.

SOARES, J.J.; SILVA, M.S. Efeito da época de plantio na produção e na ocorrência de pragas em culturas do algodoeiro (Gossypium hirsutum). Arquivos do Instituto Biológico, v.70, p.295-302, 2003.

SOUSA, M.E.C.; SANTOS, F.A.B.; WANDERLEY-TEIXEIRA, V.; TEIXEIRA, A.A.C.; SIQUEIRA, H.A.A. de; ALVES, L.C.; TORRES, J.B. Histopathology and ultrastructure of midgut of Alabama argillacea (Hübner) (Lepidoptera: Noctuidae) fed Bt-cotton. Journal of Insect Physiology, v.56, p.1913-1919, 2010.

TATE, H.R.L.; LEWIS, L.C. Evaluation of Ostrinia nubilalis (Lepidoptera: Crambidae) neonate preferences for corn and weeds in corn. Journal of Economic Entomology, v.99, p.1987-1993, 2006.

TORRES, J.B.; RUBERSON, J.R. Interactions of Bacillus thuringiensis Cry1Ac toxin in genetically engineered cotton with predatory heteropterans. Transgenic Research, v.17, p.345-354, 2008.

TORRES, J.B.; RUBERSON, J.R. Spatial and temporal dynamics of oviposition behavior of bollworm and three of its predators in $\mathrm{Bt}$ and non-Bt cotton fields. Entomologia Experimentalis Applicata, v.120, p.11-22, 2006.

TORRES, J.B.; RUBERSON, J.R.; ADANG, M.J. Expression of Bacillus thuringiensis Cry1 Ac protein in cotton plants, acquisition by pests and predators: a tritrophic analysis. Agricultural and Forest Entomology, v.8, p.191-202, 2006.

TORRES, J.B.; RUBERSON, J.R.; WHITEHOUSE, M. Transgenic cotton for sustainable pest management: a review. In: LICHTFOUSE, E. (Ed.). Organic farming, pest control and remediation of soil pollutants: sustainable agriculture reviews. Dordrecht: Springer, 2009. p.15-54.

TRAXLER, G.; GODOY-AVILA, S. Transgenic cotton in Mexico. AgBioForum, v.7, p.57-62, 2004.

ZALUCKI, M.P.; CLARKE, A.R.; MALCOLM, S.B. Ecology and behavior of first instar larval Lepidoptera. Annual Review of Entomology, v.47, p.361-393, 2002. 